\title{
ON STATISTICAL PROPERTIES OF NONLINEAR FUNCTIONALS OF RANDOM FIELDS
}

\author{
DAREEN OMARID
}

(Received 4 June 2020; first published online 18 September 2020)

2020 Mathematics subject classification: primary 60G60; secondary 35R01, 60F17, 62L20.

Keywords and phrases: reduction principle, long-range dependence, short-range dependence, noncentral limit theorems, Hermite distributions, stochastic partial differential equations, spherical random fields.

In classical statistical theory, observations are often assumed to be independent. However, in many cases this assumption may not be realistic or easily verifiable. In practical applications, researchers usually face situations in which the observations are dependent over time or space. The analysis and properties of statistical procedures substantially differ with the type of dependence between observations.

In the theory of stochastic processes and fields, covariance and spectral functions play an essential role in characterising the dependence properties. Random fields or processes are classified into two types. The first is short-range or weak dependent random fields. Covariance functions of such fields decay rapidly to zero. Also, spectral densities of weakly dependent random fields are bounded at the origin (see [6]). The second is strong or long-range dependent random fields. There are several different definitions of long-range dependence in the literature. Most of these are based on properties of second-order random processes or fields, such as variances of partial sums, the behaviour of covariances at infinity and spectral densities at the origin (see $[2,5,8]$ ). The popularity of such properties is that they are based on relatively simple characteristics that can be easily estimated from the data. The most well-known definition is that a random field is strongly dependent if its covariance function is not integrable or summable. Long-range dependence can also be defined based on singular properties of the spectral density, such as unboundedness at zero (see $[6,7]$ ).

The first two parts of the thesis concern the asymptotics of nonlinear transformations of vector random fields that take the form

$$
K_{r}=\frac{1}{C_{r}} \int_{\Delta(r)} G(\boldsymbol{\eta}(x)) d x,
$$

Thesis submitted to La Trobe University in December 2019; degree approved on 8 April 2020; principal supervisor Andriy Olenko, co-supervisor Luke Prendergast.

(C) 2020 Australian Mathematical Publishing Association Inc. 
where $\boldsymbol{\eta}(x)$, for $x \in \mathbb{R}^{d}$, is a vector random field, $G(\cdot)$ is a nonlinear function, $\Delta(r) \subset \mathbb{R}^{d}$, for $r>0$, is a bounded increasing observation window and the constant $C_{r}$ is a normalisation. The majority of results in this thesis are about different types of asymptotic behaviour of nonlinear transformations (via integral functionals or solutions of stochastic partial differential equations (SPDE), which can also be represented as different integral functionals) of scalar or vector random fields (see [6]). The main tool used to obtain the results is the spectral and correlation theory of random fields.

The reduction principles for the asymptotics of functionals $K_{r}$, when $r \rightarrow \infty$, are derived by considering two situations. In the first situation, it is assumed that all the components of $\boldsymbol{\eta}(x)=\left(\eta_{1}(x), \ldots, \eta_{m}(x)\right)^{\prime}, x \in \mathbb{R}^{d}$, possess long-range dependence properties but might have different long-range behaviour and parameters. In this case the covariance matrix $B(x)$ takes the form

$$
B(0)=\mathcal{I}, \quad B_{i j}(\|x\|)= \begin{cases}0 & \text { if } i \neq j, \\ \|x\|^{-\alpha_{j}} L_{j}(\|x\|) & \text { if } i=j,\end{cases}
$$

where $\mathcal{I}$ is the unit matrix of size $m, i, j \in\{1, \ldots, m\}, \alpha_{j}>0$ and $L_{j}(\cdot)$ are slowly varying functions at infinity. As $\alpha_{j} \in(0, d), j=1, \ldots, m$, the diagonal elements of the covariance matrix $B(\cdot)$ are not integrable functions, which corresponds to the case of long-range dependence.

In the second situation, we consider a vector random field with $m+n$ components, that is, $\boldsymbol{\eta}(x)=\left[\eta_{1}(x), \ldots, \eta_{m}(x), \eta_{m+1}(x), \ldots, \eta_{m+n}(x)\right]^{\prime}, x \in \mathbb{R}^{d}$. It is assumed that the components of $\boldsymbol{\eta}(x)$ can have different dependence structures (short- and long-range dependence). In this case the covariance matrix $B(x)$ takes the form

$$
B_{i j}(\|x\|)= \begin{cases}0 & \text { if } i \neq j, \\ \mathcal{I}_{1} \cdot\|x\|^{-\beta} L_{1}(\|x\|) & \text { if } i=j=1, \ldots, m, \beta>d, \\ \mathcal{I}_{2} \cdot\|x\|^{-\alpha} L_{2}(\|x\|) & \text { if } i=j=m+1, \ldots, m+n, \alpha<d / \kappa,\end{cases}
$$

where $\mathcal{I}, \mathcal{I}_{1}$ and $\mathcal{I}_{2}$ are unit matrices of size $m+n, m$ and $n$, respectively, $L_{i}(\|\cdot\|)$, for $i=1,2$, are slowly varying functions at infinity and $\kappa$ is the Hermite rank of the function $G(\cdot)$. Here the diagonal elements of the covariance matrix $B(\cdot)$ are integrable for the first $m$ elements of $\boldsymbol{\eta}(\cdot)$, which corresponds to the case of short-range dependence, and nonintegrable for the other elements, which corresponds to the case of long-range dependence. The reduction principle obtained shows that, contrary to the scalar case, in the vector case terms at the Hermite rank level do not necessarily determine the asymptotic behaviour. Simulation studies are conducted which support the theoretical findings. Also, we show how the results can be applied to investigate the asymptotic behaviour of Minkowski functionals of Fisher-Snedecor and Student random fields. The results are obtained under very general conditions and can be applied to study asymptotics of various statistical models and build large sample estimation theory.

In the last part of the thesis, stochastic hyperbolic diffusion equations in $\mathbb{R}^{3}$ with random initial conditions are studied. This part of the thesis considers new models 
that are simpler than the ones suggested in [1] and [3]. Their detailed analysis is provided. The equations have a physical motivation based on data obtained from cosmic microwave background radiation studies. The exact solutions to the equations are derived and given as isotropic spatial-temporal random fields. Results in this section are obtained for two scenarios. In the first scenario, it is assumed that the initial conditions are isotropic spatial-temporal spherical random fields in $\mathbb{R}^{3}$. The spectral representation and covariance structures of these fields are presented. Then, the explicit exact solutions random fields of the initial value problem are derived and analysed. In the second scenario, the restriction of the stochastic hyperbolic diffusion in $\mathbb{R}^{3}$ to the unit sphere is considered. The exact solution is derived and given in terms of a series expansion. The covariance functions, angular power spectrum and spectral representation of these fields are provided. Necessary and sufficient conditions on the spectral measure of initial conditions are provided to guarantee different types of dependence (short- or long-range dependence) of the random fields. Approximations to the exact solutions are given and analysed by truncating their series expansions. The smoothness properties of the exact solution and its approximation are studied. Also, explicit forms of upper bounds for the mean-square convergence rates of the approximation fields are given. It is demonstrated that the Hölder-type continuity of the solution random field relies on the decay of its angular power spectrum. All theoretical results are supported by numerical studies.

The main results of the thesis have been published in $[4,9,10]$.

\section{References}

[1] V. Anh, P. Broadbridge, A. Olenko and Y. Wang, 'On approximation for fractional stochastic partial differential equations on the sphere', Stoch. Env. Res. Risk. 32(9) (2018), 2585-2603.

[2] J. Beran, Y. Feng, S. Ghosh and R. Kulik, Long-Memory Processes (Springer, Heidelberg, 2013).

[3] P. Broadbridge, A. D. Kolesnik, N. Leonenko and A. Olenko, 'Random spherical hyperbolic diffusion', J. Stat. Phys. 177(5) (2019), 889-916.

[4] P. Broadbridge, A. D. Kolesnik, N. Leonenko, A. Olenko and D. Omari, 'Spherically restricted random hyperbolic diffusion', Entropy 22(2) (2020), Article ID 217.

[5] P. Doukhan, G. Oppenheim and M. S. Taqqu, Theory and Applications of Long-Range Dependence (Birkhäuser, Boston, MA, 2002).

[6] A. Ivanov and L. Leonenko, Statistical Analysis of Random Fields (Springer, Dordrecht, 1989).

[7] N. Leonenko and A. Olenko, 'Tauberian and Abelian theorems for long-range dependent random fields', Methodol. Comput. Appl. Probab. 15(4) (2013), 715-742.

[8] N. Leonenko and A. Olenko, 'Sojourn measures of Student and Fisher-Snedecor random fields', Bernoulli 20(3) (2014), 1454-1483.

[9] A. Olenko and D. Omari, 'Reduction principle for functionals of vector random fields', Methodol. Comput. Appl. Probab. 22 (2020), 573-598.

[10] A. Olenko and D. Omari, 'Reduction principle for functionals of strong-weak dependent vector random fields', Braz. J. Probab. Stat. (2020), to appear.

DAREEN OMARI, Department of Mathematics and Statistics,

La Trobe University, Melbourne, Victoria, 3086, Australia

e-mail:d.omari@latrobe.edu.au 